Retrospective Study

\title{
@ Loss of Efficacy to Spinal Cord Stimulator Therapy: Clinical Evidence and Possible Causes
}

Christopher M. Aiudi, PharmD ${ }^{1}$, Roger Y. Dunn BS, MBA², Sara M. Burns, $\mathrm{MS}^{3}$, Sarah A. Roth, BS ${ }^{3}$, Arissa Opalacz, BA ${ }^{3}$, Yi Zhang, MD, PhD, $\mathrm{MS}^{3}$, Lucy Chen, $\mathrm{MD}^{3}$, Jianren Mao, MD, $\mathrm{PhD}^{3}$, and Shihab U. Ahmed, $\mathrm{MBBS} \mathrm{MPH}^{3}$

From: ${ }^{1}$ Mayo Clinic School of Medicine, Rochester, MN; ${ }^{2}$ Tufts University School of Medicine, Boston, MA; ${ }^{3}$ Department of Anesthesia, Critical Care, and Pain Medicine, Massachusetts General Hospital and Harvard

Medical School, Boston, MA

Address Correspondence: Christopher M. Aiudi, PharmD 514 15th Ave SW Rochester, MN 55902 Email: chrisaiudi@gmail.com

Disclaimer: See pg. E1079. Conflict of interest: Each autho certifies that he or she, or a member of his or her immediate

family, has no commercial association (i.e., consultancies, stock ownership, equity interest, patent/licensing arrangements, etc.) that might pose a conflict of

interest in connection with the submitted manuscript.

Manuscript received 03-13-2017

Accepted for publication: 05-01-2017

Free full manuscript: www.painphysicianjournal.com
Background: Although spinal cord stimulation (SCS) therapy has been shown to be efficacious in various pain conditions, the ability for SCS therapy to maintain long-term efficacy has been questioned.

Objective: The purpose of this study was to investigate whether a loss of efficacy (LOE) phenomenon exists with SCS therapy and to investigate if this phenomenon is more apparent in any specific patient population.

Study Design: A retrospective, observation chart review was conducted to evaluate the patient response to SCS therapy over time.

Setting: Massachusetts General Hospital, Boston, Massachusetts.

Methods: Patients who received a SCS at the Massachusetts General Hospital, between January 1, 2002 and December 31, 2012, were invited to participate. A total of 62 patients were included in this study. Various models were created to analyze pain score changes over time using 2-tailed statistical analysis. Additionally, one-way ANOVA and Pearson's chi-square tests were used to determine if certain patient characteristics were associated with LOE.

Results: Compared to the visual analog scale (VAS) score at one month after device implantation pain scores increased 1.95 points after 2 years $(95 \% \mathrm{Cl}: 1.06$ to $2.84, P=<0.001)$. There were no significant differences in baseline characteristics between the groups of patients who did and did not lose efficacy of their therapy. However, those who experienced LOE had a baseline SCS therapy VAS score 3.09 points lower than those who did not $(95 \% \mathrm{Cl}: 1.69$ to $4.48, P=<0.001)$.

Limitations: This study had several limitations including the retrospective nature of its design, confounders to VAS scores, small sample size, missing data points, and the evaluation of only conventional, low-frequency SCS therapy.

Conclusions: Patients who received a SCS had a significant increase in VAS scores over time. Our data did not show any baseline patient characteristic that helped predict LOE. However, patients who have significant baseline response to therapy may be more likely to experience LOE.

Key words: Spinal cord stimulation, chronic pain, retrospective study, low frequency electrical stimulation, efficacy, chronic pain therapy

Pain Physician 2017; 20:E1073-E1080 he gate-control theory, proposed by Melzack and Wall in 1965, describes how stimulated large afferent fibers of the dorsal column are able to block ascending pain signals within the spinal cord (1). In 1967, spinal cord stimulator (SCS) therapy brought the gate-control theory into the clinical realm when Shealy used the device and demonstrated significant pain relief without distortion of other sensory or motor functions in a cancer patient (2). Since then, clinical studies have shown the efficacy of 
SCS therapy for several indications such as failed back surgery syndrome, chronic low back pain, and complex regional pain syndrome (3-10).

Although SCS therapy has been shown to be efficacious in various pain conditions, the ability for SCS therapy to maintain long-term efficacy has been questioned. In fact, in clinical practice it has been recognized that the efficacy of SCS therapy diminishes with time (10-15).

The purpose of this study was to investigate if SCS therapy loses efficacy over time. The primary outcome of interest was the visual analog scale (VAS) score, which is a widely used scale for measuring pain (16-17). We hypothesized that VAS scores increase with SCS therapy over time due to loss of efficacy (LOE). The secondary outcome of interest was to assess if any baseline patient characteristics predisposed patients to being more likely to develop LOE of SCS therapy.

\section{Methods}

This study was approved by the Massachusetts General Hospital Institutional Review Board, and all of the patients provided prior consent for the use of their medical records for research purposes.

\section{Study Participants}

Patients who received a permanent SCS at the Massachusetts General Hospital were eligible to participate in this retrospective cohort study. We included patients aged 18 years and older who received a permanent SCS between January 1, 2002 and December 31, 2012. We excluded patients who were unable to be contacted, and thus could not provide consent for the study. Sixty-two patients met the inclusion criteria and were included in this study

\section{Data Collection}

Baseline patient demographics and clinical characteristics were extracted from electronic medical records at the time of SCS implantation and follow-up appointments. The data extracted at the time of SCS implantation including patient demographics, medical history, pain history (i.e., indications for SCS, location and character of pain, VAS score prior to implantation), and procedural characteristics.

Follow-up data were extracted, if available, at predefined time intervals post-implantation: 1 month, 3 months, 6 months, 1 year, and then annually until either device removal, loss to follow-up, or patient records reached the current treatment periods. The data extracted at follow-up visits included patient demographics (work and disability allowance status) and pain characteristics (VAS score and pain description).

\section{Defined Measurements}

\section{Baseline SCS VAS Score}

The baseline SCS VAS score was defined as the VAS score recorded at the one-month post-implant followup visit or as the first follow-up visit if the patient missed their initial appointments.

\section{$\angle O E$}

LOE was defined as an increase in VAS score equal to or greater than $20 \mathrm{~mm}$ (2 points) from the baseline VAS score on SCS therapy for at least 2 consecutive patient follow-up visits. A change in $20 \mathrm{~mm}$ (2 points) on the VAS has been validated as the difference where patients can clinically distinguish different levels of pain, the "minimum clinical important difference (MCID)" (18-22). An increased VAS score for 2 consecutive patient follow-up visits was used in order to define LOE to ensure suboptimal pain coverage persisted and was not a result of acute events or temporary device malfunction.

\section{Statistical Analysis}

All analyses were performed using SAS Version 9.4 (SAS Institute, Inc., Cary, NC) and R statistical software (RStudio, Version 3.2.2; R Foundation for Statistical Computing, Vienne, Austria). Patient demographics and characteristics are reported in Table 1. Descriptive statistics were presented as mean (SD) or frequency counts (\%), as appropriate. Since this was a retrospective cohort study, no a priori statistical power calculation was performed. The analysis was conducted on all available data.

To examine whether individuals experienced LOE to SCS therapy, several models were constructed. To accommodate the varying number of repeated measures across the patients, random intercepts models were used to analyze VAS changes over time. The models were adjusted for the following prespecified covariates: baseline pain, age, body mass index (BMI), gender, disability, and depression. To optimize statistical power, 2 models were utilized. The first model takes into account only the first 2 years of follow-up data and the second model assesses the full 12 years of follow-up data. All hypothesis testing was 2 -tailed with significance interpreted as $P<0.05$. Additionally, one- 
Table 1. Overall Baseline Patient Characteristics

\begin{tabular}{|c|c|c|c|c|}
\hline & $\begin{array}{c}\text { Overall Patient } \\
\text { Population } \\
(\mathbf{n}=\mathbf{6 4})\end{array}$ & $\begin{array}{c}\text { Patients Who Did NOT } \\
\text { Experience LOE } \\
(n=42)\end{array}$ & $\begin{array}{c}\text { Patients Who Did } \\
\text { Experience LOE } \\
(n=20)\end{array}$ & $P$-Value \\
\hline Age (yrs), mean (SD) & $49.77(13.0)$ & $49.98(12.6)$ & $49.35(13.9)$ & 0.86 \\
\hline BMI, mean (SD) & $29.67(7.0)$ & $29.23(7.2)$ & $30.61(6.5)$ & 0.49 \\
\hline VAS score, mean (SD) & $6.52(2.2)$ & $6.60(2.3)$ & $6.33(1.8)$ & 0.67 \\
\hline Gender, n (\%) & & & & 0.67 \\
\hline Female & $33(53.2 \%)$ & $21(50.0 \%)$ & $12(60.0 \%)$ & 0.46 \\
\hline Male & $29(46.8 \%)$ & $21(50.0 \%)$ & $8(40.0 \%)$ & \\
\hline \multicolumn{5}{|l|}{$\begin{array}{l}\text { Medication Use for Depression, } \mathrm{n} \\
(\%)\end{array}$} \\
\hline No & $34(55.7 \%)$ & $24(58.5 \%)$ & $10(50.0 \%)$ & 0.53 \\
\hline Yes & $27(44.3 \%)$ & $17(41.5 \%)$ & $10(50.0 \%)$ & \\
\hline \multicolumn{5}{|l|}{ Disability, n (\%) } \\
\hline No & $36(58.1 \%)$ & $23(54.8 \%)$ & $13(65.0 \%)$ & 0.45 \\
\hline Yes & $26(41.9 \%)$ & $19(45.2 \%)$ & $7(35.0 \%)$ & \\
\hline \multicolumn{5}{|l|}{ Pain Time, n (\%) } \\
\hline Intermittent & $21(35.6 \%)$ & $13(32.5 \%)$ & $8(42.1 \%)$ & 0.73 \\
\hline Constant & $19(32.2 \%)$ & $14(35.0 \%)$ & $5(26.3 \%)$ & \\
\hline Unspecified & $19(32.2 \%)$ & $13(32.5 \%)$ & $6(31.6 \%)$ & \\
\hline \multicolumn{5}{|l|}{ Pain Characteristic, n (\%) } \\
\hline Neuropathic & $32(54.2 \%)$ & $21(52.5 \%)$ & $11(57.9 \%)$ & 0.74 \\
\hline Nociceptive & $8(13.6 \%)$ & $5(12.5 \%)$ & $3(15.8 \%)$ & \\
\hline Unspecified Radicular Pain & $8(13.6 \%)$ & $5(12.5 \%)$ & $3(15.8 \%)$ & \\
\hline Neuropathic and Nociceptive & $11(18.6 \%)$ & $9(22.5 \%)$ & $2(10.5 \%)$ & \\
\hline
\end{tabular}

LOE = loss of efficacy; $\mathrm{SD}$ = standard deviation; VAS = visual analog scale

way ANOVA tests and Pearson's chi-square tests were used to assess the differences in baseline characteristics between patients who experienced LOE and those who did not experience LOE in the device.

\section{Results}

\section{Baseline Demographic Characteristics}

During the prespecified time period, 340 patients received a SCS at the Massachusetts General Hospital. Enrollment for the study is described in Fig. 1. The patient population consisted of 62 patients, balanced between genders (53.2\% female, $46.8 \%$ male) with a mean age of 49.8 years and an average BMI of 29.7. The average VAS score prior to the time of SCS trial/implant was 6.52. Almost half of the patient population had a history of depression (43.3\%) and were on disability allowance $(41.9 \%)$. The indications for SCS implantation included failed back surgery syndrome (43.6\%), complex regional pain syndrome $(25.8 \%)$, peripheral neuropathy refractory to conservative measures (8.1\%), and chronic lumbar or cervical radiculopathy refractory to conservative measures (8.1\%). Several rare indications included piriformis pain syndrome, occipital headaches, brachial plexus neuritis, post-traumatic neuropathic pain syndrome, Dercum's disease (lipomatosis dolorosa), and uncontrolled pain secondary to multiple myeloma.

The remaining overall baseline patient characteristics for our study population can be found in Table 1.

\section{Pain Scores over Time}

Prior to implantation of the SCS, the average VAS score was 6.52 points $(S D=2.2)$. As a result of a decreasing sample size as the time variable continued (see Fig. 2), 2 models were created to report VAS changes over time. Model 1 was created to analyze only the first 2 years of follow-up data, whereas Model 2 assessed all follow-up VAS scores.

From Model 1, a substantial increase in the mean VAS score over time was observed (see Fig. 3A). Com- 


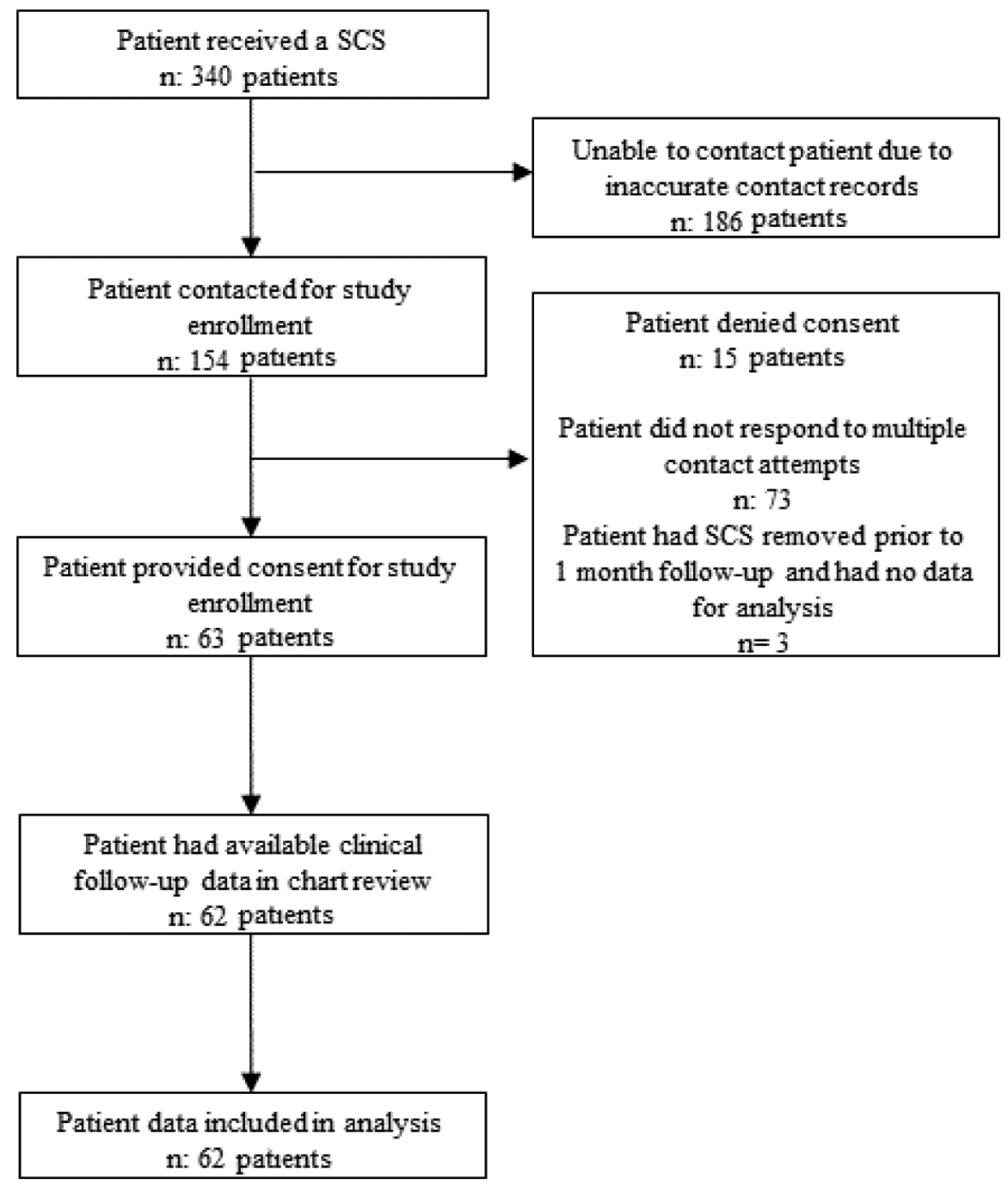

Fig. 1. Patient enrollment flow diagram.

pared to the VAS score at one month (baseline SCS therapy VAS score), pain scores increased 0.62 VAS points at the 3 -month follow-up $(95 \% \mathrm{Cl}:-0.25$ to 1.49 , $P=0.157), 1.21$ points at the 6 -month follow-up $(95 \%$ $\mathrm{Cl}: 0.34$ to $2.07, P=0.007), 1.37$ points at the one-year follow-up ( $95 \% \mathrm{Cl}: 0.51$ to $2.22, P=0.002$ ), and 1.95 points at the 2 -year follow-up $(95 \% \mathrm{Cl}: 1.06$ to $2.84, P$ $=<0.001)$. This trend supports the hypothesis that LOE to SCS therapy may develop over time. In Model 2 (Fig. $3 \mathrm{~B})$, there was a VAS score increase of 0.01 points on average with every month that passed over the 12-year span ( $95 \% \mathrm{Cl}: 0.00$ to $0.02, P=0.017)$.

\section{Associations between LOE and Patient Characteristics or Pain Characteristics}

An additional model, Model 3, was created to assess the relationships between LOE and baseline patient characteristics. For the purpose of this study, we artificially dichotomized patients into 2 groups: those who had LOE (as defined previously) and those who did not have LOE. Of note, $65.5 \%$ of patients did not experience LOE with their SCS therapy. In Model 3, the interaction between LOE and time was evaluated (see Fig. 4). The baseline efficacy of SCS therapy differed significantly between the LOE group and the non-LOE 


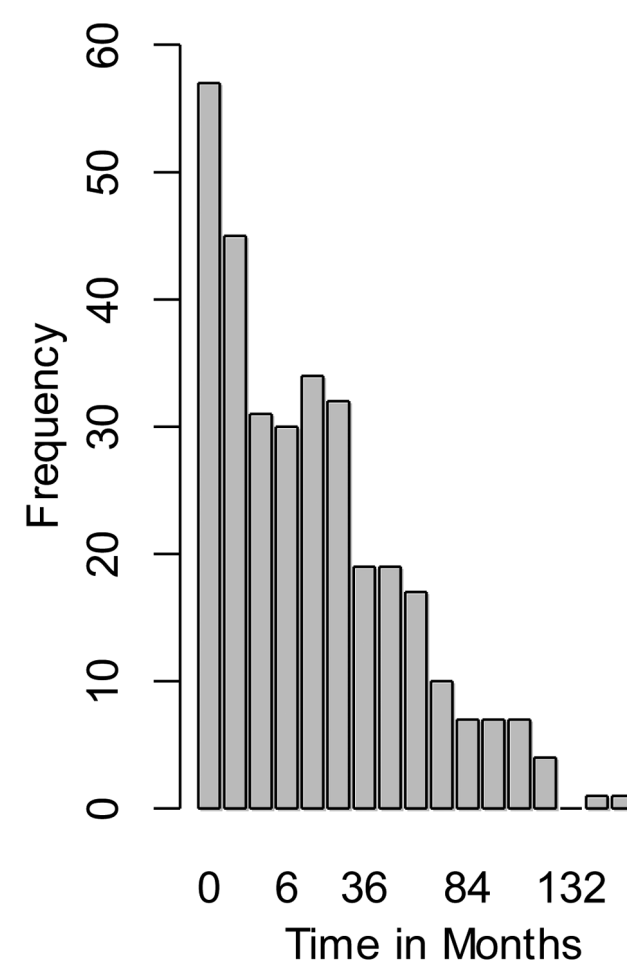

Fig. 2. Sample size over time.

${ }^{\star}$ Frequency is defined as the number of observed VAS scores (sample size) reported at each time-point.

group. Those who eventually experienced LOE had a baseline SCS VAS score 3.09 points lower than those who did not $(95 \% \mathrm{Cl}: 1.69$ to $4.48, P=<0.001)$, even though the trial/pre-implantation VAS scores were only 0.27 points different $(P=0.67)$ between the groups. However, at the one-year follow-up, those who experienced LOE had a VAS score of 1.57 points higher than those who did not ( $95 \% \mathrm{Cl}: 0.07$ to $3.06, P=0.040)$.

The interaction between LOE and baseline demographics and pain characteristics of the 2 groups was analyzed. There were no significant differences in baseline characteristics between the group of patients who experienced LOE of SCS therapy and the group of patients who did not (see Table 1).

\section{Discussion}

This retrospective cohort study suggests patients experience LOE to SCS therapy. VAS scores continually increased as more time progressed from SCS implantation, with a significant increase in VAS scores starting at 6 months post-implantation. Patients who eventually
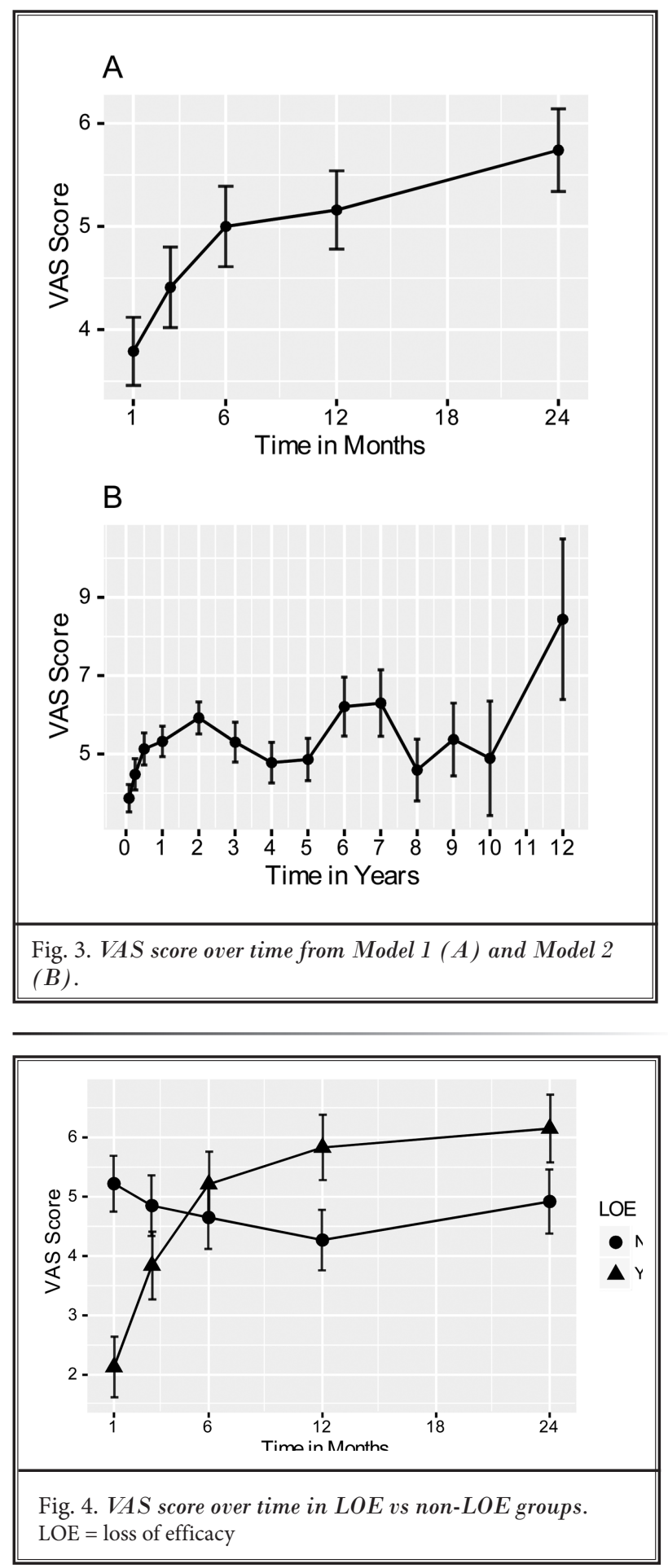

experienced LOE of their SCS therapy initially experienced significantly more pain relief compared to those who do not experience LOE. However, this pain relief 
did not persist and pain scores became significantly higher than those without LOE within one year. There were no significant differences in baseline characteristics to predict which patients will eventually experience LOE of their SCS therapy.

Previous studies have demonstrated that effective pain control with SCS decreases with time $(5,10-15)$. Results from an earlier systematic review demonstrated at one year, $62 \%$ of patients had successful pain relief but at 5 and 10 years after SCS implantation, the success rate fell to $53 \%$ and $35 \%$ respectively (13). In a similar study, patients reported significantly higher 30- and 48-month pain scores compared to their 24-month pain scores after the initiation of SCS therapy (14). More recently, a prospective study of 36 patients demonstrated an overall decrease in VAS scores with SCS but observed a linear increase in the VAS score after 1 and 2 years of follow-up $(P=0.03)(10)$. However, VAS scores were still significantly lower than pre-SCS therapy. Interestingly, previous randomized controlled trials have demonstrated a significant drop-off in SCS response at 6 months post-implantation, however those who maintained response remained relatively stable after the 6-month period $(4,7)$. Findings from this current study support not only a decrease in effective pain control with time (Fig. 3A, 3B), but also a stable pain control pattern after the 6-month follow-up period (Fig. 4) as previously described. While this study supports previous results, it also describes a pattern of pain scores within the first 6 months which might help predict which patients experience LOE of SCS therapy.

When comparing the LOE and non-LOE cohorts, there was a significant difference in baseline SCS efficacy in the LOE cohort even though the pre-implant VAS scores did not vary significantly. Since the baseline characteristics between the 2 groups are statistically similar, we can only speculate causes for this difference such as a possible larger placebo effect, differences in patient expectations, or underlying psychological characteristics such as catastrophizing. Other possible mechanisms for this increase in VAS scores include underlying disease progression, change in paresthesia coverage with time due to device migration or malfunction, changes in microenvironment surrounding the active electrodes leading to the development of high impedances, the development of new pain conditions or clinical condition associated with chronic pain, or functional improvement leading to activities that increase pain.

The data from this study demonstrated a trend which may allow clinicians to have a better indication of which patients may eventually experience LOE of SCS therapy. Patients who experienced LOE had a significant initial response to SCS therapy, but then shortly began to observe a rapid increase in the VAS score over one year. The data suggests if a patient experiences this rapid and drastic improvement in their pain control with SCS therapy, they may be more likely to eventually have LOE of SCS therapy. Therefore, if this trend is seen, the use of alternative pain management strategies may be useful to supplement SCS therapy to treat their increasing pain severity. Conversely, patients who do not have a rapid response to SCS therapy are less likely to eventually experience LOE of SCS therapy and experience longer periods of decreased pain before returning to their pre-SCS VAS score.

This study had several limitations including the retrospective nature of its design, confounders to VAS scores, small sample size, missing data-points, and the evaluation of only conventional, low-frequency SCS therapy. The retrospective nature of this study did not allow control for several confounders including multiple underlying pain conditions and the progression of the primary condition. VAS scores were obtained from medical records during SCS therapy follow-up appointments but this may not entirely represent the pain relating to the target condition the SCS therapy is treating. Since many patients with chronic pain have multiple pain conditions, the accurate rating of VAS scores for a single pain entity is difficult. Worsening of the underlying pain condition is another confounder since this often is of a gradual nature, which could be unnoticed by the patient. Therefore, we could not rule out the issue of disease progression causing increased VAS scores. Furthermore, VAS scores are highly variable and can be influenced by many aspects of treatment, such as changes in pain medication use and other treatment modalities, which were not accurately recorded during follow-up data. A small sample size also limited the length of our statistical models. Patients had to be contacted via mail and/or phone to be informed about this study and for staff to obtain consent. Many patients who received a SCS were unable to be contacted because of an incorrect address, disconnected phone number, study staff was unable to make direct contact (after 3 unreturned voicemails and mailings), or the patient was deceased; 15 patients also denied consent. As a result, the patient cohort was not large enough to extend our primary model to a follow-up period of $5-6$ years or more as other SCS therapy efficacy analyses have done $(13,15)$. Another limitation of our study 
included missing data-points. The data were collected at predefined time intervals post-SCS implantation. The majority of patients had at least one missing data-point in their chart review. Some patients may not return for follow-up appointments due to LOE, adequate pain coverage, or length of follow-up between appointments. While there are several methods to handle missing data such as multiple imputation (MI) or last observation carried forward (LOCF), we deemed these methods inappropriate for our analysis due to lack of sample size, which could lead to misinterpretation of the data (23). Therefore, we chose a follow-up period of 24 months for our primary model to ensure adequate sample size to make meaningful inferences. While Model 2 (Fig. 3B) does interpret data up to 12 years of follow-up, the declining sample size of data-points beyond the 24 months used for the primary model may be clinically irrelevant. Finally, this study only included conventional, low-frequency, paresthesia-based SCS therapy. Newer high-energy sub-perception SCS devices and programs, such as high-frequency $(10 \mathrm{kHz})$ SCS devices and "burst" stimulation, were not evaluated in this study as a result of the predefined time period. Although, the newer systems have been shown to maintain efficacy at 24 months, long-term data is not available $(7,24)$.

This study was one of the first studies to directly analyze loss of SCS therapy efficacy over time based on VAS scores as the primary outcome. Patients who received SCS therapy had a significant increase in VAS scores as time progressed. Our data did not show any differences in baseline patient characteristics that could help predict if a patient may or may not experience LOE of SCS therapy. However, our data from a single large academic institution suggests that patients who have a significant response to SCS therapy immediately after implantation may be more likely to eventually develop LOE to the therapy. While the study design created several limitations, this study does provide data that could have clinical implications on pain management. Therefore, it should be utilized as a hypothesisgenerating foundation to encourage future research to better understand the possible etiologies to this LOE phenomenon.

\section{Funding Sources}

This work was supported by a grant from the Medical Student Anesthesia Research Fellowship Program (MSAR) though the Foundation for Anesthesia Education and Research (FAER). The FAER program provided funding for cost of living and travel to the academic site (Massachusetts General Hospital, Boston MA) for research experience and 2016 American Society of Anesthesia Conference for abstract presentation.

\section{Authorship Statement}

All individuals involved in this research were involved in the authorship of the manuscript. Accordingly, they are included in the authorship component on the first page.

\section{References}

1. Melzack R, Wall PD. Pain mechanism: A new theory. Science 1965; 150:971-979.

2. Shealy CN, Mortimer JT, Reswick JB. Electrical inhibition of pain by stimulation of the dorsal columns: Preliminary clinical report. Anesth Analg 1967; 46:489-491.

3. Kemler MA, Barendse GA, van Kleef $M$, de Vet HC, Rijks CP, Furnée CA, van den Wildenberg FA. Spinal cord stimulation in patients with chronic reflex sympathetic dystrophy. N Engl J Med 2000; 343:618-624.

4. Kumar K, Taylor RS, Jacques L, Eldabe S, Meglio M, Molet J, Thomson S, O'Callaghan J, Eisenberg E, Milbouw G, Suchser E, Fortini G, Richardson J, North RB. The effects of spinal cord stimulation in neuropathic pain are sustained: A 24- month follow-up of the prospective randomized controlled multicenter trial of the effectiveness of spinal cord stimulation. Neurosurgery 2008; 63:762-770.

5. Kumar K, Hunter G, Demeria D. Spinal cord stimulation in treatment of chronic benign pain: Challenges in the treatment planning and present status, a 22-year experience. Neurosurgery 2006; 58:481-496.

6. North RB, Kidd DH, Farrokhi F, Piantadosi SA. Spinal cord stimulation versus repeated lumbosacral spine surgery from chronic pain: A randomized, controlled trial. Neurosurgery 2005; 56:98-106.

7. Kapural L, Yu C, Doust MW, Gliner BE,
Vallejo R, Sitzman BT, Amirdelfan K, Morgan DM, Brown LL, Yearwood TL, Bundschu R, Burton AW, Yang T, Benyamin $\mathrm{R}$, Burgher $\mathrm{AH}$. Novel 10-kHz high-frequency therapy (HFio therapy) is superior to traditional low-frequency spinal cord stimulation for the treatment of chronic back and leg pain: The SENZA-RCT randomized controlled trial. Anesthesiology 2015; 123:851-860.

8. Deer TR, Mekhail N, Provenzano D, Pope J, Krames E, Leong M, Levy RM, Abejon D, Buchser E, Burton A, Buvanendran A, Candido K, Caraway D, Cousins M, DeJongste M, Diwan S, Eldabe S, Gatzinsky K, Foreman RD, Hayek S, Kim P, Kinfe T, Kloth D, Kumar K, Rizvi S, Lad SP, Liem L, Linderoth B, Mackey S, McDowell G, McRoberts P, Poree L, Prager J, Raso L, 
Rauck R, Russo M, Simpson B, Slavin $K$, Staats $P$, Stanton-Hicks M, Verrills $P$, Wellington J, Williams K, North R; Neuromodulation Appropriateness Consensus Committee. The appropriate use of neurostimulation of the spinal cord and peripheral nervous system for the treatment of chronic pain and ischemic diseases: The Neuromodulation Appropriateness Consensus Committee. Neuromodulation 2014; 17:515-550.

9. Deer TR, Skaribas IM, Haider N, Salmon J, Kim C, Nelson C, Tracy J, Espinet A, Lininger TE, Tiso R, Archacki MA, Washburn SN. Effectiveness of cervical spinal cord stimulation for the management of chronic pain. Neuromodulation 2014; 17:265-271.

10. Forouzanfar T, Kemler MA, Weber W, Kessels AG, Kleef M. Spinal cord stimulation in complex regional pain syndrome: Cervical and lumbar devices are comparably effective. $\mathrm{Br}$ ] Anaesth 2004; 92:348-353.

11. Mann SA, Sparkes E, Duarte RV, Raphael $\mathrm{JH}$. Attrition with spinal cord stimulation. $\mathrm{Br}$ J Neurosurg 2015; 29:823-828.

12. Kemler MA, de Vet HC, Barendse GA, van den Wildenberg $F A$, van Kleef $M$. Effect of spinal cord stimulation for chronic complex regional pain syndrome Type I: Five-year final follow-up of patients in a randomized controlled trial. J Neurosurg 2008; 108:292-298.

13. Turner JA, Loeser JD, Bell KG. Spinal cord stimulation for chronic low back pain: A systematic literature synthesis. Neurosurgery 1995; 37:1088-1096.

14. Aló KM, Redko V, Charnov J. Four year follow-up of dual electrode spinal cord stimulation for chronic pain. Neuromodulation 2002; 5:79-88.

15. Turner JA, Loeser JD, Deyo RA, Sanders SB. Spinal cord stimulation for patients with failed back surgery syndrome or complex regional pain syndrome: A systematic review of effectiveness and complications. Pain 2004; 108:137-147.

16. Jensen MP, Karoly P, Braver S. The measurement of clinical pain intensity: A comparison of six methods. Pain 1986; 27:117-126.

17. Jensen MP, McFarland CA. Increasing the reliability and validity of pain intensity measurement in chronic pain patients. Pain 1993; 55:195-203.

18. Farrar JT, Young JP Jr., LaMoureaux L, Werth JL, Poole RM. Clinical importance of changes in chronic pain intensity measured on an 11-point numerical rating scale. Pain 2001; 94:149-158.

19. Dworkin RH, Turk DC, Wyrwich KW, Beaton D, Cleeland CS, Farrar JT, Haythornthwaite JA, Jensen MP, Kerns RD, Ader DN, Brandenburg N, Burke LB,
Cella D, Chandler J, Cowan P, Dimitrova R, Dionne R, Hertz S, Jadad AR, Katz NP, Kehlet H, Kramer LD, Manning DC, McCormick C, McDermott MP, McQuay HJ, Patel S, Porter L, Quessy S, Rappaport BA, Rauschkolb C, Revicki DA, Rothman M, Schmader KE, Stacey BR, Stauffer JW, von Stein T, White RE, Witter J, Zavisic $S$. Interpreting the clinical importance of treatment outcomes in chronic pain clinical trials: IMMPACT recommendations. J Pain 2008; 9:105-121.

20. Todd KH, Funk KG, Funk JP, Bonacci R. Clinical significance of reported changes in pain severity. Ann Emerg Med 1996; 27:485-489.

21. Gallagher EJ, Liebman M, Bijur PE. Prospective validation of clinically important changes in pain severity measured on a visual analog scale. Ann Emerg Med 2001; 38:633-638.

22. Bird SB, Dickson EW. Clinically significant changes in pain along the visual analog scale. Ann Emerg Med 2001; 38:639-643.

23. Kim Y. Missing data handling in chronic pain trials. J Biopharm Stat 2011; 21:311-325.

24. De Ridder D, Vanneste S, Plazier M, van der Loo E, MenovskyT. Burst spinal cord stimulation: Toward paresthesia-free pain suppression. Neurosurgery 2010; 66:986-990. 